\title{
Percepción de la soledad en los adultos mayores de la localidad de Molas, Yucatán
}

\section{Perception of loneliness in older adults in the locality of Molas, Yucatan}

Francelia M. Kú

Martha A. Lope

Marianeli García

Estefany G. Carrillo

Universidad Autónoma de Yucatán (UADY)

\section{Resumen}

La presente investigación tuvo como objetivo explorar si está presente el sentimiento de soledad, en adultos mayores de Molas, Yucatán, así como conocer la percepción y significado que tiene para ellos. Se consideraron aspectos como la familia, relaciones interpersonales, cultura, sentimientos, emociones, situación laboral como posibles factores involucrados en la percepción del sentimiento de soledad. Se realizaron entrevistas a 25 personas para la recolección de información. En los resultados obtenidos, se encontró que el sentimiento de soledad no se encuentra presente en los participantes debido a la cultura de la localidad y la cercanía de sus familiares y amigos.

Palabras clave: vejez, soledad, percepción, cultura, sentimientos.

\begin{abstract}
The research aimed to explore if the feeling of loneliness is present in older adults in Molas, Yucatan, as well as to know their perception and meaning for it. Aspects such as family, interpersonal relations, culture, feelings, emotions, work situation were considered as possible factors involved in their perception of the feeling of loneliness. We interviewed 25 people for the collection of information. The results obtained show that the feeling of loneliness is not present in the participants due to the culture of the locality and the closeness of their relatives and friends.
\end{abstract}

Keywords: old age, loneliness, perception, culture, feelings.

Nota del autor

Francelia M. Kú, Facultad de Psicología, Universidad de Yucatán (UADY).

La correspondencia en relación con este artículo debe dirigirse a Francelia M. Kú, Facultad de Psicología, UADY, calle 60 491-A, colonia Centro, C.P. 97000, Mérida, Yucatán, México.

Dirección electrónica: PercepcionSoledad@gmail.com 
En la actualidad se enfrentan dilemas teóricos relacionados con las condiciones cambiantes y complejas del contexto histórico y social; por ejemplo, el Instituto Nacional de las Personas Adultas Mayores (INAPAN, 2010) menciona que se conoce una condición de desigualdad, con repercusiones en la vida individual, familiar y social de los adultos mayores e, incluso implica la discriminación hacia la etapa de la vejez.

De lo anterior, se establece que es necesario explorar la cultura actual, conocer lo que piensan los adultos de la tercera edad, cómo perciben la soledad. Al respecto, en un estudio de Peplau y Perlman (como se citó en Montero, \& Sánchez, 2001) se menciona que la soledad es un aspecto subjetivo, que puede percibirse de diversas maneras según la persona que la experimente; desde el aislamiento, hasta la ausencia de apoyo social.

La presente investigación se centra en conocer más sobre los adultos mayores que viven la etapa de la vejez, a través de las experiencias propias que guían a explorar sus sentimientos, emociones, historia, familia y amigos, para conocer qué tan cerca experimentan un sentimiento de soledad, en caso de experimentarla o no, cómo la perciben, cómo la viven, los procesos que se enfrentan y aclarar los aspectos que influyen en esta etapa para entenderla de la manera como la describen.

Por el contacto directo, a través de conversaciones informales, surgió la inquietud de describir la percepción de la soledad en los adultos mayores, ya que como mencionan
Expósito y Moya (2000), la soledad no es característica únicamente de quienes la experimentan, sino un proceso dinámico $\mathrm{y}$ complejo que va más allá del ámbito privado, hasta convertirse en un problema social. El presente estudio puede considerarse relevante, puesto que la soledad es subjetiva (Peplau y Perlman en Montero, \& Sánchez, 2001), y la información fue recolectada a través de la experiencia de vida de los adultos mayores de la localidad de Molas, lo cual permite explorar y analizar los aspectos que están involucrados en la experiencia de la soledad.

La investigación proporciona opciones para que se realicen investigaciones en las que se tomen en cuenta las percepciones sobre la soledad de los jóvenes, adultos o adultos mayores de otros lugares con el fin de determinar si la perspectiva difiere según el lugar, la edad, las experiencias y la cultura.

\section{Marco referencial}

Según Peplau y Perlam (como se citó en Montero, \& Sánchez, 2001), la soledad es concebida desde diferentes visiones, dado que en la mayoría de las definiciones existentes, se encuentran las siguientes descripciones: condición de aislamiento físico, falta de relaciones interpersonales y una experiencia nada placentera para las personas que la experimentan.

\section{Historia y cultura}

La concepción de la vejez ha cambiado por el contexto histórico y/o cultural, por ejemplo, hoy en día los adultos mayores son ubicados en las 
escalas menos valoradas de la sociedad (Robles, 2006).

\section{Sentimientos y emociones}

De acuerdo con el informe del NorthAmerican Nursing Diagnosis Association(NANDA, 2001), los factores que provocan aislamiento social son la ausencia de relaciones interpersonales satisfactorias, nivel socioeconómico bajo, enfermedades, deterioro físico y/o mental. Por otro lado, para Pochintesta (2012), uno de los sentimientos asociados con el envejecimiento es el miedo a la muerte propia, perder autonomía, independencia, sufrir deterioro corporal, es decir, sentirse vulnerables.

\section{Familia}

En México, de acuerdo con el informe del Instituto Nacional de Estadística, Geografía e Informática (INEGI, 2014), las personas de 60 años y más que se estimaron para 2014, 31.5\%, están en una etapa de prevejez (60 a 64 años); $41.1 \%$ se encuentran en una vejez funcional (65 a 74 años); $12.3 \%$ vive en una vejez plena (75 a 79 años) y $15.1 \%$ transita por una vejez avanzada (80 años y más). Al relacionar lo anterior con lo planteado por Márquez (como se citó en Quintanar, 2010), a saber, en México, especialistas de la salud consideran que la familia es el grupo social fundamental para ayudar al adulto mayor a desempeñar sus roles e integrarse a la sociedad como sujeto de su propio desarrollo. Sin embargo, al retomar lo dicho por Ussel et al., (2001), la familia también puede ser la principal causa del aislamiento social.

\section{Factores que influyen en la soledad}

La Dispersión de los hijos es un factor con influencia en la soledad, ya que la familia es muy importante en la etapa de la vejez; cuando esta relación con los hijos es positiva, los adultos mayores poseen una gran motivación y tranquilidad. La cercanía o la distancia entre la vivienda de los padres y los hijos influyen en esta relación, pues, a pesar de ser buena, los adultos mayores pueden sentirse solos si los hijos viven lejos de ellos. Esta condición genera angustia en su vida, y se incrementa cuando se tiene algún problema de salud que dificulta gozar de una vida plena y autónoma (Ussel et al., 2001).

En cuanto a las Relaciones Interpersonales, como factor con efectos en la soledad, se sabe que la falta de atención y diálogo puede ocasionar que las personas se perciban menos integradas en el medio social (Ramos, \& Salinas, 2010). Esta edad le concede una gran importancia al hecho de mantener una identidad y estar activos e integrados en diversos espacios y grupos.

La viudez como influyente en la soledad logra desencadenar diversas consecuencias: puede representar la transición a la etapa de la vejez, la disolución parcial o completa de la familia original (Tovar, Pérez-Cárdenas, \& Infante como se citó en Montes de Oca, 2011), incluso se experimenta de diferentes maneras dependiendo del contexto histórico, cultural, el número de hijos, número de años vividos como pareja, género de la persona que vive, recursos 
económicos y el afecto de los hijos hacia el padre que aún vive (Montes de Oca, 2011).

Asimismo, la actividad laboral es factor de influencia en la soledad puesto que las personas mayores dejan de trabajar por diversas causas, como los problemas de salud, sin embargo, existe otro grupo que sigue laborando para satisfacer sus necesidades, cubrir gastos médicos, mantener una vida digna, etc. Según Montoya y Montes (como se citó en Montoya, \& Montes, 2009), con énfasis en lo dicho por Ramos y Salinas (2010), una de las principales razones por las que los adultos mayores siguen trabajando es la necesidad de mantenerse activos, para ellos el trabajo no es sólo una actividad, sino representa el desarrollo y mantenimiento de una identidad.

Por otra parte, la jubilación representa una etapa importante en la vejez. Las pensiones y jubilaciones están frecuentemente asociadas con las actividades laborales que los individuos con 60 años o más hayan experimentado a lo largo de su vida (Montoya, \& Montes, 2009). Para muchos es sinónimo de ser viejo e improductivo. Silvestre, Solé, Pérez y Jódar (como se citó en Ramos, Salinas, Colín, Mora, \& Maldonado, 2012) exponen tres formas de ver a la jubilación: un proceso o evento, un rol social y una crisis.

En cuanto a la salud, Rice (como se citó en Quintanar, 2010), señaló que la vejez comienza a los 65 años y se caracteriza por un declive gradual del funcionamiento de todos los sistemas corporales, y se convierte en uno de los factores que limitan la calidad de vida.

\section{Método}

Se utilizó como medio el enfoque cualitativo en la investigación y la fenomenología hermenéutica como el método de investigación para poder conocer los significados que los individuos dan a su experiencia.

\section{Escenario}

Se trabajó en la localidad de Molas, pertenecienteal municipio de Mérida, en el estado de Yucatán; cuenta con 777, 615 habitantes, de los cuales la población masculina de 60 años o más es de 36, 382 y la población femenina de 60 años o más es de 47, 527 personas según, INEGI (2010). El trabajo de campo se realizó en los hogares de los participantes seleccionados de manera aleatoria.

\section{Participantes}

De acuerdo a Craig (2006), el periodo de la vejez comienza a los 60 años de edad, aproximadamente, y puede abarcar 40 años. En tal contexto, se trabajó con hombres y mujeres de 60 años como edad mínima y 92 años como edad máxima. Todos los participantes viven en la localidad de Molas, Yucatán.

\section{Técnicas y/o instrumentos de recolección de datos}

Se utilizó la técnica de entrevista, consiste en una reunión para conversar e intercambiar información entre el entrevistador y el entrevistado (Martorell, \& González, 1997). Se 
llevaron a cabo entrevistas semiestructuradas, donde los temas de interés fueron la percepción de la soledad, historia y cultura, sentimientos y emociones, familia, así como los factores que influyen en la soledad, es decir, dispersión de los hijos, relaciones interpersonales, viudez, actividad laboral y jubilación, salud, dependencia económica, apoyo del gobierno, relaciones interpersonales, y dependencia física y emocional. La aplicación de esta técnica permitió conocer de manera cercana, y desde la voz de los participantes voluntarios, qué es lo que rodea, piensa y la manera en la que se expresa el adulto mayor de Molas, Yucatán. En sí conocer la percepción que se tiene de la experiencia de la soledad y los aspectos involucrados en la misma.

El dispositivo utilizado para la recopilación de información fue una grabadora digital marca Sony modelo ICD PX 333D y una hoja impresa con la guía de entrevista. Como parte de las consideraciones éticas, se llevó a cabo un consentimiento informado, en el cual las personas aceptaban participar en el trabajo de investigación y permitían grabar la entrevista en audio; en caso de sentirse incómodas, podrían retirarse de la entrevista. Además se recalcó la total confidencialidad y anonimato.

\section{Procedimiento}

Primero se realizó un mapeo físico, a través de un registro obtenido en internet; el objetivo fue conocer la manera en la que está conformada la localidad. Posteriormente, se dividió el mapa en tres zonas para la realización las entrevistas. Asimismo, se llevó a cabo un diagnóstico social para conocer cómo es la dinámica de la población.

En el primer contacto con los participantes, se les explicó el fin de la investigación y se les informó acerca del anonimato y confidencialidad de los datos proporcionados, resaltando los fines educativos que poseían. Al aceptar, los participantes firmaron un consentimiento informado, donde daban la autorización de participar y grabar la entrevista en audio, video y/o imágenes. Cada entrevista duró entre 45 minutos y/o hora y media. Una vez realizadas las 25 entrevistas, se procedió a transcribirlas para llevar a cabo un análisis de los datos, resaltando los puntos y temas de interés.

\section{Resultados}

Se trabajó con personas de ambos sexos, la mayoría mujeres; con diversos estados civiles, aunque la mayoría eran casados, seguida de viudos y separados. El rango de edad fue de 60 años a 92 años, siendo 61 años la edad predominante. El nivel académico fue principalmente de primaria. A continuación, se describe a detalle la información proporcionada dentro de cada categoría: Percepción de la Soledad; Historia y Cultura; Sentimientos y Emociones; Familia; Factores que influyen en la soledad, 
entre los cuales se encuentran Dispersión de los Hijos, Relaciones Interpersonales, Viudez, Actividad Laboral y Jubilación, además de Salud.

Para comenzar, la Percepción de la Soledad para los participantes significa estar aislados físicamente $\mathrm{y} / \mathrm{o}$ no compartir el hogar con otras personas, a pesar de que tienen como vecinos a familiares. La opinión acerca de la experiencia de la soledad para algunos era algo desagradable, otros la consideraban cómoda, debido a que dedicaban el tiempo a su cuidado personal o al cuidado de sus animales y/o de sus plantas. En cuanto a la Historia y Cultura, al describir las actividades diarias que realizan, la mayoría incluyó las actividades del hogar: cocinar, limpiar, lavar, barrer, regar las plantas, desyerbar, ejemplo de esto es la aseveración siguiente:

Me levanto, voy al baño vengo a mi cocina, después de que desayunemos a trabajar, preparar comida, lavar trastes, luego mi cocina, luego al almuerzo, lavar trastes y otra vez a la cocina, en la tarde descanso un ratito (Mujer, entrevista, septiembre del 2015).

Por otra parte, los participantes consideran que la localidad es tranquila, pacífica y que puede ser habitada sin problemas; igualmente, describen como buena la relación entre los vecinos, mencionaron que se apoyan entre ellos cuando necesitan algo e, incluso, cuando un problema se presenta:
Pues nuestra comunidad es muy pacífica, mayormente somos gentes humildes, gente que se lleva, yo me llevo bien con todos... aquí nos conocemos todos (Mujer, entrevista, octubre del 2015).

Sin embargo, no todos los participantes están de acuerdo con esta descripción, otros la perciben conflictiva, como quien narra lo siguiente:

Mala, mucho maleante, mucho pleito, es como se dicen... pueblo chico, infierno grande (Mujer, entrevista, septiembre del 2015).

Los participantes mencionaron que su interacción con los jóvenes es diferente a la que se efectuaba en años anteriores, consideraron que se debe a la pérdida de valores, entre ellos al respeto, expresado, por ejemplo, en el hecho de saludar a los adultos o ayudarlos cuando lo necesiten:

Hay ahorita muchachitos que ya no respetan... Cuando nosotros crecimos, cualquier persona grande que cruce a lado de uno, le dan los buenos días, buenas tardes o noches. Era el respeto que así teníamos con las personas grandes, pero ahora, no, ahora los muchachitos, o sea, ni aunque te conozcan te hablan, nada, es eso lo que pasa ahora, no, antes no era así, no es igual, ya no es igual (Mujer, entrevista, septiembre del 2015).

En cuanto a Sentimientos y Emociones, describieron sus emociones como elementos contribuyentes a su bienestar, donde la alegría 
es predominante en la mayoría de la vida de los adultos mayores. Sin embargo, los participantes mencionaron que sus emociones pueden llegar a ser negativas; el enojo es una de las más importantes:

No tengo ninguna tristeza, no hay ninguna... Alegría sí (Hombre, entrevista, octubre del 2015).

Yo me enojo mucho, sí, yo sí, cualquier cosa me molesta, pero yo digo que es por lo de mi depresión (Mujer, entrevista, noviembre del 2015).

Incluso, para los participantes, hay situaciones que modifican su emoción, ya sea por algún problema familiar, enfermedad, o al pensar en la situación económica en la que se encuentran, además de las imposibilidades físicas.

Hoy mi sentimiento es feliz, muy feliz (se ríe), lo que lo cambiaría es que mis hijos o alguien le pase algo o hagan algo que me enoje, pero depende (Mujer, entrevista, octubre del 2015).

En tal contexto, señalaron dos factores que les ayudan a lograr su estabilidad emocional: el primero es un factor espiritual, la religión.

Sé que Dios está conmigo y me digo ¿por qué estás triste? Si él siempre está a mi lado protegiéndome y ya me siento más tranquila asi (Mujer, entrevista, octubre del 2015).

En cuanto al factor de Familia, se considera uno de los factores más importantes en relación con sus sentimientos y emociones; indican que les ayuda en la estabilidad emocional. Todos los participantes se consideran importantes, ya que se sienten apoyados y comprendidos por la familia y una parte fundamental de ella.

Yo creo que yo soy el pilar de mis 4 hijos (Hombre, entrevista, septiembre del 2015).

Cada familia está conformada de manera distinta, la mayoría son extensas, catorce es el máximo de integrantes en el hogar, como es el caso de un participante; en contraste, el mínimo lo representa a otra familia conformada por dos personas.

En esta casa vivimos entre tres familias, somos como 12, somos bastantes

(Mujer, entrevista, octubre del 2015).

Los participantes definieron la relación con sus hijos de manera tranquila y cercana, en la que interactúan de manera recíproca.

En cuanto Factores que Influyen en la Soledad, se consideró la Dispersión de los Hijos. La relación padre(s)- hijo(s) es positiva, expresaron que ellos siguen pendientes de sus necesidades y cuidados, esta característica se encuentra influida por la cercanía de la vivienda entre ellos, aunque en caso de ser lejana éstos también mantienen una buena comunicación.

Sigo teniendo una relación cercana con ellos, vienen a verme y todo eso (Hombre, entrevista, septiembre del 2015).

En cuanto a las Relaciones Interpersonales como factor de influencia en la soledad, se dio a conocer que los grupos de amigos están conformados por los vecinos, con quienes platican o realizan alguna actividad; un participante mencionó ser asocial, en palabras específicas él expresó: 
No, es que toda mi vida he sido antisocial, pero tengo amigos, pero que yo me reúna y tenga una rutina, hace 4 o 5 años sí iba a un café a platicar con mis amigos, pero ahora ya no (Hombre, entrevista, octubre del 2015).

La mayoría de las personas indicó que realiza actividades de ocio, como costurar, bordar, pintar, practicar deporte, leer, platicar, hacer ejercicio, etc., para entretenerse, sentirse útiles, además de contribuir al desarrollo de su bienestar físico, emocional y social.

En cuanto a la Viudez, se encontró que algunos de los adultos mayores han experimentado la pérdida del cónyuge. Los participantes expresaron que fue una etapa difícil y triste, sin embargo, el apoyo de su familia les ayudó a sobreponerse ante la situación. Consideran que han aceptado o se han resignado ante la pérdida, viven tranquilos y en paz, recordando los momentos especiales que vivieron junto a su pareja.

En cuanto a la Actividad Laboral, existen participantes que trabajan gracias a su buena condición física $\mathrm{y}$ salud. Otros realizan actividades como vender productos, tejidos, artesanías, entre otros, para generar ingresos económicos, debido a que no reciben pensión. De igual manera, hay personas que dependen de un familiar; mientras que otros, otras son el sostén de su familia. Asimismo, algunos participantes participan en programas que les dan apoyo económico, de gran ayuda para ellos.

Finalmente, sobre la Salud, la mayoría de los participantes presenta problemas de salud física, no obstante, expresaron tener motivación para seguir adelante, mejorarse, compartir tiempo con la familia y realizar actividades diarias. Entre las enfermedades se encuentran chicungunya, problemas cardíacos, reumatismo, diabetes, osteoartritis, azúcar elevada o baja, presión alta o baja, asma, parálisis facial, ceguera, entre otros.

Pues es eso, es mis rodillas que me duelen... padezco de la presión, padezco del corazón, pero estoy controlado... Estoy en el seguro popular (Mujer, entrevista, noviembre del 2015).

\section{Discusión}

Definir la soledad no es algo fácil, como bien lo señalan Peplau y Perlam (como se citó en Montero, \& Sánchez, 2001), la soledad es concebida desde diferentes visiones. En la presente investigación se confirmó lo anterior, en este caso se pudo observar que para los adultos de la tercera edad que habitan en Molas, el aislamiento físico la representa, pero ellos no lo padecen. Es por eso que la percepción de ellos concuerda con los autores ya mencionados, puesto que la soledad varía de acuerdo con la experiencia de cada persona y población.

Con base en lo anterior, se toma en cuenta la Historia y Cultura, ya que, para los participantes, el concepto acerca de la vejez efectivamente va transformándose: perciben un cambio en el trato hacia ellos en comparación con el pasado, 
similitud con lo planteado por Robles (2006), pues sienten que son ubicados en las escalas menos valoradas de la sociedad; de igual manera, señalaron que existía una disminución del respeto hacia otros adultos mayores.

En cuanto a los Sentimientos y Emociones de los participantes, dio a conocer un gran equilibrio emocional, donde la felicidad fue la emoción más destacada, lo cual difiere de lo planteado por Pochintesta (2012), quien menciona que el miedo puede presentarse en esta etapa, debido al deterioro corporal que experimentan; sin embargo, los participantes a pesar de sufrir esos cambios físicos, aceptan esta etapa de su vida, saben que los cambios seguirán avanzando; Lo que les ayuda a mantenerse estables emocionalmente es la religión y los fuertes vínculos que mantienen con la familia.

Hablar de la Familia como categoría y la Dispersión de los Hijos como factor que influye en la soledad, la íntima relación temática nos lleva a conjuntar ambos elementos. Principalmente es relevante diferenciar el vivir solos con el sentirse solos; muchas personas a pesar de vivir junto a su familia, se sentían solas, en tanto algunas que vivían solas, no se sentían de esa manera, debido a las relaciones interpersonales que mantienen.

Cabe mencionar que en su mayoría, los participantes viven con su cónyuge, hijos o parientes, condición que contrasta con lo registrado por el INEGI (2010), he aquí la diferencia cultural de datos específicos en comparación con los datos generales que proporciona el instituto. Los hijos normalmente viven con sus padres o cerca de ellos; incluso, al no vivir con ellos, los participantes mantienen contacto estrecho; lo cual demuestra que no están dispersos y que la familia es una pieza fundamental en su vida, los apoya en su equilibrio emocional, tal como lo plantean Domínguez, Espín y Bayorre (2001). Sin embargo, en opinión de Ussel y colaboradores (2001), cuando las relaciones familiares son hostiles, aparecen sentimientos de tristeza, pero no la soledad, esto se debe a las relaciones interpersonales y a la seguridad emocional que tienen. Por lo tanto, encontramos que la teoría revisada coincide en varios aspectos con nuestra investigación, ya que los adultos mayores de Molas dejan en claro la visión de la familia unida que conservan como una parte central de sus vidas.

En cuanto al tema de Relaciones Interpersonales, se determinó que es un factor que influye en la percepción de la soledad, y concuerda con lo expresado por Ramos y Salinas (2010), puesto que los participantes viven relaciones agradables con sus vecinos y gente de la localidad, lo cual permite que se sientan integrados en el medio social, y éste sea una fuente de apoyo. Por otro lado, en lo relativo a la Viudez, la información recabada contrasta con lo expresado por Pérez (2007), quien resalta la propensión al suicidio en personas que experimentan la muerte de su pareja. En la presente investigación, quienes han experimentado esta situación no expresaron 
deseos de morir, sino aceptaron que sintieron tristeza, pero la compañía de sus hijos y amigos contribuyó a aceptar lo sucedido y seguir con sus vidas de la mejor manera posible.

Acerca de los temas como Actividad laboral y Jubilación (los cuales se decidió conjuntar), los testimonios concuerdan con lo expresado por Márquez (como se citó en Quintanar, 2010), ya que las instituciones y organizaciones pueden ser de gran apoyo para los adultos jubilados o que no trabajan; aunque en este caso, no todos tienen esa posibilidad, muchos adultos mayores no cuentan con el apoyo del gobierno, y se ven en la necesidad de realizar alguna actividad para generar ingresos; otros mas reciben apoyo de su familia, como también lo han registrado Montoya y Montes (2009), dado que esta etapa de la vida disminuye la generación de ingresos económicos por los problemas de salud y físicos.

Finalmente, respecto a la Salud, se encontró concordancia con Rice (como se citó en Quintanar, 2010), en que hay un declive gradual del funcionamiento de todos los sistemas corporales en las personas de la tercera edad, empero, los participantes de este estudio han aceptado cada enfermedad o cambio que se les presenta, ejecutan los cuidados pertinentes y realizan lo mejor posible sus actividades diarias.

Esta investigación cuenta con información exteriorizada por hombres y mujeres de tercera edad; en la localidad, ambos son tomados en cuenta para la toma de decisiones $\mathrm{y}$, a pesar de la presencia de alguna enfermedad física, se esfuerzan para seguir con sus labores, por ende, resulta difícil que se les excluya de actividades físicas productivas.

Se concluye que, en la localidad de Molas, Yucatán, la soledad no se experimenta debido al fuerte papel que desempeñan las relaciones familiares. Además, los factores ambientales, culturales y sociales influyen en la percepción que los participantes tienen acerca de la soledad. Los resultados presentados aportan información nueva, diferente y complementaria a la teoría expuesta en años anteriores, demuestran que no es una regla que la soledad esté presenta en la etapa de la vejez, asimismo, rompen con la imagen de la vejez como una de las etapas más difíciles y con menos posibilidades de disfrutar de la vida.

Debido al enfoque utilizado se pudo conocer la manera en la que viven, sienten, piensan los participantes y las diversas percepciones que tienen acerca de la soledad. Sin embargo, se presentan limitaciones: el tamaño de la localidad, así como la imposibilidad de entrevistar a todos los adultos mayores que viven en ello. La información que se presenta puede utilizarse para realizar un contraste con otras investigaciones, así como aportar información para algún proyecto enfocado en los adultos mayores de Molas, Yucatán.

Este trabajoha sido una experiencia totalmente diferente, con un resultado inesperado que contrasta con la visión de otras investigaciones, donde la soledad es una característica que la mayoría de las personas mayores experimentan y que muchos consideran una norma en la vejez. 


\section{Referencias}

Craig, G. (2006). Desarrollo Psicológico. México: Pearson.

Domínguez, T., Espín, A., \& Bayorre, H. (2001). Caracterización de las relaciones familiares del anciano. Revista cubana de Medicina General Integral, (21), 1-2. Recuperado de http://bvs.sld.cu/revistas/mgi/vol17_5_01/ mgi02501.pdf

Expósito, F., \& Moya, M. (2000). Percepción de la soledad. Psicothema, 12(4), 123-125.

Instituto Nacional de Estadística y Geografía. (2010). Datos de población. Recuperado de http://www.inegi.org.mx/est/contenidos/ proyectos/ccpv/default.aspx

Instituto Nacional de Estadística y Geografía. (2014). Datos de población. Recuperado de http://www.inegi.org.mx/saladeprensa/ aproposito/2014/adultos0.pdf

Instituto Nacional de las Personas Adultas Mayores. (2010). Por una Cultura del Envejecimiento. México, DC: Autor.

Martorell, M., \& González, R. (1997). Entrevista y Consejo Psicológico. Madrid: Síntesis.

Montero, M., \& Sánchez. J. (2001). La soledad como fenómeno psicológico: un análisis conceptual. Salud Mental, 24(1), 11-27.

Montes de Oca, V. (2011). Viudez, soledad y sexualidad en la vejez: mecanismos de afrontamiento y superación. Recuperado de http://seminarioenvejecimiento.unam.mx/ Publicaciones/articulos/viudez_soledad_sex. pdf
Montoya, B., \& Montes, H. (2009). Situación laboral de la población adulta mayor en el Estado de México. Papeles de POBLACIÓN, 15(59), 193-237.

North American Nursing Diagnosis Association. (2001). Diagnósticos Enfermeros: Definiciones y Clasificación (2001-2002). Madrid: Harcourt.

Pérez, M. (2007). El duelo en el anciano. En W. Astudillo, A. Ispiuza, A. Orbegozo., \& M. Pérez (Eds.), Acompañamiento en el duelo y medicina paliativa (pp. 89-103). San Sebastián: Sociedad Vasca de Cuidados Paliativos.

Pochintesta, P. (2012). Antropología de la subjetividad. Recuperado de http://www. antropologiadelasubjetividad.com/images/ trabajos/paula_pochintesta.pdf

Quintanar, A. (2010). Ánalisis en la calidad de vida en adultos mayores del municipio de Tetepango, Hidalgo: A través del instrumento Whoqol-Bref. (Tesis de licenciatura). Actopan, Hidalgo. Universidad Autónoma del Estado de Hidalgo. Recuperado de http:// www.uaeh.edu.mx/nuestro_alumnado/esc sup/actopan/licenciatura/Analisis\%20de $\% 20$ la\%20calidad\%20de\%20vida.pdf

Ramos, J., \& Salinas, R., (2010). Vejez y apoyo social. Revista de Educación y Desarrollo, (15), 69-76. Recuperado de http://www. redadultosmayores.com.ar/Material\%20 $2013 / \mathrm{Nacionales} \% 20 \mathrm{Mexico} / 6 \% 20$ vejez $\% 20 y \% 20$ apoyo $\% 20$ social.pdf 
Ramos, J., Salinas, R., Colín, G., Mora, A., \& Maldonado, I. (2012). Representaciones sobre la jubilación y la vejez en personas mayores jubiladas y pensionadas de la Ciudad de Morelia, México. Revista Educación y Desarrollo, (23), 71-79

Robles, L. (2006). La vejez: nuevos actores, relaciones sociales y demandas políticas. Relaciones, 105. 27. 141-175

Ussel, J., López, J., Díaz, M., Alemán, C., Trinidad, A., \& Castón, P. (2001). La soledad en las personas mayores: Influencias Personales, Familiares y Sociales. Instituto de Migraciones y Servicios Sociales (IMSERSO). Recuperado de file://C:/ Users/home/Downloads/La-soledad-en-laspersonas-mayores.pdf.

Recibido el 16 de mayo de 2016 Revisado el 22 de julio de 2016 Aceptado el 25 de julio de 2016 